\title{
Prevalence of infections with Clostridium difficile on potential pathology groups
}

\author{
Traian TACHE ${ }^{*}{ }^{1}$ Răzvan CHIRICĂ, ${ }^{1}$ Marius-Daniel RADU, $, 2,3$ Gabriela GEGIU, ${ }^{3}$ \\ and Sorin RUGINA $\breve{A}^{1}$ \\ ${ }^{1}$ Faculty of Medicine, Ovidius University of Constanta, Romania \\ ${ }^{2}$ Faculty of Natural and Agricultural Sciences, Ovidius University of Constanta, Romania \\ ${ }^{3}$ Faculty of Pharmacy, Ovidius University of Constanta, Romania
}

\begin{abstract}
Enterotoxins produced by Clostridium difficile cause a series of biochemical and immunological manifestations in the cascade leading to alteration of the enterocitus cytoskeleton, intestinal inflammation and diarrhea that can greatly impair the patient's biological status. The genome of the Clostridium difficile bacterium shows a series of evolutionary adaptations that can give it a high degree of resistance or adaptability to many known pharmacological classes. Changing the diversity of intestinal microbiota induced by the use of antibiotics creates a favorable environment from all points of view for Clostridium difficile spore activity. The theme addresses in an original way but related to the epidemiological studies presented in the literature a correlative aspect between the pathological group and the infection with Clostridium difficile. From the data presented, there is a direct correlation between Clostridium difficile infection and the use of antibiotic therapy as a curative or preventive treatment. Gastrointestinal and neurological pathologies, due to the use of curative but also preventive antibiotic therapy, are at increased risk for the installation of Clostridium difficile infection. The study presented may be a first step in raising awareness of the rational use of antibiotics and avoiding non-assisted community antibiotic therapy.
\end{abstract}

Keywords: Clostridium difficile; resistance forms; pathology groups.

\section{Introduction}

Clostridium difficile is an anaerobic gram-positive bacterium that presents in its biological cycle and the form of resistance enhancement. Clostridium difficile colonizes the digestive tract of the immune-treated or immunosuppressive immune system [1]. Of the total number of patients with antibiotic-associated diarrhea, $15-30 \%$ is the main cause of Clostridium difficile infection, and 3-8\% has an increased potential for developing severe pathologies such as toxic megacolon, perforation of colon with peritonitis and septic shock [1]. The pathophysiology of infection is strictly associated with the production of two pathogenic enterotoxins with cytotoxic potential on intestinal epithelial cells causing pseudomembranous colitis [2, 3].

Enterotoxins produced by Clostridium difficile cause a series of biochemical and immunological manifestations in the cascade leading to alteration of the enterocitus cytoskeleton, intestinal inflammation and diarrhea that can greatly impair the patient's biological status [4].

In vitro experimental models have shown that there are correlations between the use of a type of antibiotic and the installation of Clostridium difficile infection and the results appear to correlate very well with the clinical risk of Clostridium difficile infection [5].

Clinical practice in the treatment schedule for Clostridium difficile infection takes into account all the clinical manifestations presented by the patient but requires the first phase of discontinuation of the antibiotic treatment that facilitates colonization of the intestine with Clostridium difficile $[6,7]$. Metronidazole is used in mild to moderate Clostridium difficile infections, while vancomycin is the most commonly used pharmacological form in the treatment of moderate but also severe infections with specific enterotoxic manifestations [8]. This pharmacological scheme that includes metronidazole and vancomycin shows a high rate of inefficiency in the treatment of Clostridium difficile infection. Inadequacy is based on a number of factors including the biology of the individual, the drug alternative to the treatment of infection and recurrence with Clostridium difficile; narrow-spectrum antibiotic fidaxomicin, broad spectrum antibiotic rifaximin, which exhibits local pharmacological character without absorption in the intestine and nitazoxanide, a pharmacological combination quoted in the literature [9 - 13].

The pharmacological mechanism of action of metronidazole is aimed at the specific denaturing of the bacterial genetic material and the death of the bacterium. Metronidazole passively passes inside the bacteria where, at the cytoplasmic level, the prodrug is converted into a radical with a nitroso free group that interacts nonspecifically with bacterial DNA and causes the blocking of the organization and reorganization of the genetic material, which leads to the denaturation of the DNA and cell death [14, 15].

The genome of the Clostridium difficile bacterium shows a series of evolutionary adaptations that can give

\footnotetext{
* Corresponding author.E-mail address: tache_traian@yahoo.com (Traian Tache)
} 
it a high degree of resistance or adaptability to many known pharmacological classes. Summarizing, metronidazole inhibits nucleic acid synthesis of anaerobic bacterial cell. However, epidemiological studies report an increase in the resistance of Clostridium difficile to the pharmacological effect of metronidazole. Another aspect of the administration of metronidazole to Clostridium difficile infection is the neurotoxic effect manifested on the patient's nervous tissue as reported in recently published studies [16]. As mentioned above, fidaxomicin is currently widely used in the treatment of Clostridium difficile infection, especially for recurrent cases. Fidaxomicin was approved in Germany and the US in 2011 for use in the treatment of Clostridium difficile infections in adult patients [17].

The treatment of bacterial cells and forms of resistance with different classes of antibiotics aims at eliminating the bacterium with pathogenic potential without harming the physiology of the patient. Thus, the outcome of therapy largely depends on the antibiotic target and its mechanism of action. In multiple recurrence cases, although it is used in standard antibiotic therapy, fecal microbial transplantation should be considered [18, 19]. Human intestinal microbiota is a highly complex microbial community in a biochemical interaction, but genetics is also rigorous. However, unnecessary antibiotic consumption often reduces the diversity of intestinal microflora and negatively influences the normal genetic and biochemical balance of intestinal and colonial microbial colonies. The microflora of patients with relapsing infection with Clostridium difficile is characterized by a significant decrease in diversity in the normal and non-pathogenic colonial colonization but also in the large intestine [20].

\section{Experimental}

The data were taken from the Public Health Directorate of Constanta and the reporting and processing of medical information took effect for the time period mentioned 08.02.2015 - 04.06.2016. Only the medical information of patients aged over 18 who live in rural and urban areas was included in the study. The study protocol was signed and approved by the institutional evaluation commission and the Ethics Committee of the Public Health Department of Constanta.

The data has been processed in compliance with the legal protocol on the use of personal data. During hospitalization, patients were actively pursued to collect data on the evolution of diarrheal episodes, the onset of medical complications, and death. Diagnosis of Clostridium difficile infection was confirmed by the determination of toxin $\mathrm{A}$ and $\mathrm{B}$ and glutamate dehydrogenase by chemiluminescence in faeces.

The diagnosed cases were organized into five pathology groups as follows: gastrointestinal and hepatic pathologies (Gastroenterology), renal pathologies (Nephrology), contagious-infectious pathologies (Infectious diseases), genital pathology (ObstetricsGynecology) and surgical pathologies (Surgery).

The pathology groups were chosen according to the degree of antibiotic use.

\section{Results and discussion}

The data are summarized in Fig. 1 and Table 1.

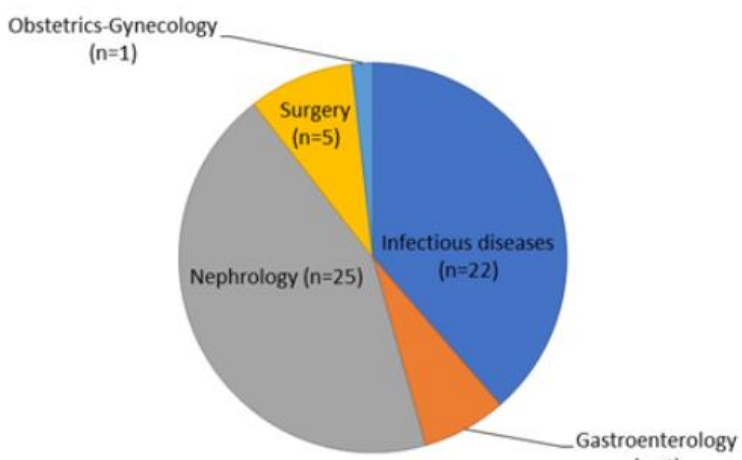

$(n=4)$

Figure 1. Case's number of Clostridium difficile infection, organized by pathological groups ( $\mathrm{n}=$ number of patients).

From the presented figure it appears that certain pathological groups have a higher incidence for Clostridium difficile infection using the current use of antibiotic therapy combined with anti-inflammatory.

Table 1. Case's number of Clostridium difficile infection with sepsis associated with antibiotic therapy, organized by pathological groups ( $\mathrm{n}=$ number of patients).

\begin{tabular}{|c|c|}
\hline $\begin{array}{c}\text { Total number of } \\
\text { patients }\end{array}$ & Sepsis \\
\hline \multicolumn{2}{|c|}{ Infectious diseases } \\
\hline $\mathrm{n}=22$ & $\mathrm{n}=2$ \\
\hline $\mathrm{n}=4 \quad \mathrm{nastroenterology}$ \\
\hline $\mathrm{n}=25 \quad$ Nephrology \\
\hline Obstetrics-Gynecology \\
\hline $\mathrm{n}=1$ \\
$\mathrm{n}=5$ \\
\hline Surgery \\
\hline
\end{tabular}

From the data presented, the use of antibiotic therapy in pathological cases leads to a physiological alteration of the patient with severe complications. The infectious response is dependent on the antibiotic-induced pharmacological mechanism.

Using antibiotics in biomedical practice also requires a number of precautions. Clostridium difficile infection frequently occurs as a result of long-term antibiotic treatment without regard to the need for pathology or the biological condition of the individual. Another factor that plays a particularly important role in Clostridium difficile infection is self-induced or socially recommended, not medical, antibiotic therapy. Testing for Clostridium difficile infection should target high-risk patients with specific symptoms. The use of most unjustified antibiotics alters the balance of intestinal microflora, creating an extremely good niche for the colonization of Clostridium difficile bacteria or its sporulated forms. Changing the diversity of intestinal microbiota, induced by antibiotic use, creates a favorable environment for all Clostridium difficile spores to enter into activity. Changing the normal intestinal flora may have many causes, by which: cytostatic and laxative administration, inflammation and dissection tube neoplasms, nonspecific 
nutrition and poor quality, use of long-acting antacids, immunosuppression and surgical intervention. Thus, we identify a relatively large number of patients with predisposition to Clostridium difficile infection. The theme addresses in an original way but related to the epidemiological studies presented in the literature a correlative aspect between the pathological group and the infection with Clostridium difficile. From the data presented, there is a direct correlation between Clostridium difficile infection and the use of antibiotic therapy as a curative or preventive treatment. In the gastroenterological and nephrological clinic pacing, often single antibiotics are used or in different combinations that can damage the balance of intestinal flora and the result is colonization by Clostridium difficile. The data presented refer to the importance of correct administration of antibiotics by pathogens and the analysis of antibiotic-induced clinical damage to the biology of an immunosuppressed patient or in a pathological condition. Patients with recent exposure to antibiotics require strict monitoring, they are susceptible to Clostridium difficile infection that can fulminate and induce the death of the patient.

\section{Conclusions}

Infection with Clostridium difficile continues to remain a challenge for modern clinical research, which relates to the mechanisms of action of antibiotics on the bacterial cell. The study shows a clear association between Clostridium difficile infection and various pathology groups. Gastrointestinal and neurological pathologies, due to the use of curative but also preventive antibiotic therapy, are at increased risk for the installation of Clostridium difficile infection. The study presented may be a first step in raising awareness of the rational use of antibiotics and avoiding non-assisted community antibiotic therapy.

\section{Conflict of interest}

Authors declare no conflict of interest.

\section{References}

[1]. P.H.V. Saavedra, L. Huang. F. Ghazavi, S. Kourula, T.V. Berghe, N. Takahashi, P. Vandenabeele, L. Mohamed, Apoptosis of intestinal epithelial cells restricts Clostridium difficile infection in a model of pseudomembranous colitis, Nature Communications 9 (2018) 4846. DOI: 10.1038/s41467-018-07386-5

[2]. S.A. Kuehne, S.T. Cartman, J.T. Heap, M.L. Kelly, A. Cockayne, N.P. Minton, The role of toxin A and toxin B in Clostridium difficile infection, Nature 467 (2010) 711-713. DOI: 10.1038/nature09397

[3]. D. Lyras, J.R. O'Connor, P.M. Howarth, S.P. Sambol, G.P. Carter, T. Phumoonna, R. Poon, V. Adams, G. Vedantam, S. Johnson, D.N. Gerding, J.I. Rood, Toxin B is essential for virulence of Clostridium difficile, Nature 458 (2009) 1176-1179. DOI: 10.1038/nature07822

[4]. W.K. Smits, D. Lyras, D.B. Lacy, M.H. Wilcox, E.J. Kuijper, Clostridium difficile infection, Nature
Reviews Disease Primers 2 (2016) 16020. DOI: 10.1038/nrdp.2016.20

[5]. J. Freeman, F.J. O'Neill, M.H. Wilcox, Effects of cefotaxime and desacetylcefotaxime upon Clostridium difficile proliferation and toxin production in a triple-stage chemostat model of the human gut, Journal of Antimicrobial Chemotherapy 52 (2003) 96-102. DOI: 10.1093/jac/dkg267

[6]. A.N. Ananthakrishnan, Clostridium difficile infection: epidemiology, risk factors and management, Nature Reviews Gastroenterology \& Hepatology 8 (2011) 17-26. DOI: 10.1038/nrgastro.2010.190

[7]. M. Kachrimanidou, N. Malisiovas, Clostridium difficile infection: a comprehensive review, Critical Reviews in Microbiology 37 (2011) 178-87. DOI: 10.3109/1040841X.2011.556598

[8]. A.M. Jarrad, T. Karoli, A. Debnath, C.Y. Tay, J.X. Huang, G. Kaeslin, A.G. Elliott, Y. Miyamoto, S. Ramu, A.M. Kavanagh, J. Zuegg, L. Eckmann, M.A. Blaskovich, M.A. Cooper, Metronidazoletriazole conjugates: activity against Clostridium difficile and parasites, European Journal of Medicinal Chemistry 101 (2015) 96-102. DOI: 10.1016/j.ejmech.2015.06.019

[9]. K.Z. Vardakas, K.A. Polyzos, K. Patouni, P.I. Rafailidis, G. Samonis, M.E. Falagas, Treatment failure and recurrence of Clostridium difficile infection following treatment with vancomycin or metronidazole: a systematic review of the evidence, International Journal of Antimicrobial $\begin{array}{llll}\text { Agents } & 40 & \text { (2012) } & 1-8 .\end{array}$ 10.1016/j.ijantimicag.2012.01.004

[10]. I.R. Poxton, Fidaxomicin: a new macrocyclic, RNA polymerase-inhibiting antibiotic for the treatment of Clostridium difficile infections, Future Microbiology 5 (2010) 539-48. DOI: 10.2217/fmb.10.20.

[11]. F. Chaparro-Rojas, K.M. Mullane, Emerging therapies for Clostridium difficile infection - focus on fidaxomicin, Infection and Drug Resistance 6 (2013) 41-53. DOI: 10.2147/IDR.S24434

[12]. E.C. Oldfield IV, E.C. Oldfield III, D.A. Johnson, Clinical update for the diagnosis and treatment of Clostridium difficile infection, World Journal of Gastrointestinal Pharmacology and Therapeutics 5 (2014) 1-26. DOI: 10.4292/wjgpt.v5.i1.1

[13]. D.M. Musher, N. Logan, A.M. Bressler, D.P. Johnson, J.F. Rossignol, Nitazoxanide versus vancomycin in Clostridium difficile infection: a randomized, double-blind study, Clinical Infectious Diseases 48 (2009) 41-6. DOI: 10.1086/596552

[14]. K.M. Land, P.J. Johnson, Molecular basis of metronidazole resistance in pathogenic bacteria and protozoa, Drug Resistance Updates 2 (1999) 289294. DOI: 10.1054/drup.1999.0104

[15]. S. Lofmark, C. Edlund, C.E. Nord, Metronidazole is still the drug of choice for treatment of anaerobic infections, Clinical Infectious Diseases 50 (2010) S16-23. DOI: 10.1086/647939

[16]. A. Kuriyama, J.L. Jackson, A. Doi, T. Kamiya, Metronidazole-induced central nervous system toxicity: A systematic review. Clinical 
Neuropharmacology 34 (2011) 241-247. DOI: 10.1097/WNF.0b013e3182334b35.

[17]. S. Maa, A. Otto, D. Albrecht, K. Riedel, A. Trautwein-Schult, D. Becher, Proteomic signatures of Clostridium difficile stressed with metronidazole, vancomycin, or fidaxomicin, Cells 7 (2018) 213. DOI: $10.3390 /$ cells 7110213

[18]. C.M. Surawicz, L.J. Brandt, D.G. Binion, A.N. Ananthakrishnan, S.R. Curry, P.H. Gilligan, L.V. McFarland, M. Mellow, B.S. Zuckerbraun, Guidelines for diagnosis, treatment, and prevention of Clostridium difficile infections, American Journal of Gastroenterology 108 (2013) 478-498. DOI: 10.1038/ajg.2013.4

[19]. T.G. Gweon, K.J. Lee, D.H. Kang, S.S. Park, K.H. Kim, H.J. Seong, T.H. Ban, S.J. Moon, J.S.
Kim, S.W. Kim, A case of toxic megacolon caused by Clostridium difficile infection and treated with fecal microbiota transplantation, Gut and Liver 9 (2015) 247-250. DOI: 10.5009/gnl14152

[20]. J.Y. Chang, D.A. Antonopoulos, A. Kalra, A. Tonelli, W.T. Khalife, T.M. Schmidt, V.B. Young, Decreased diversity of the fecal microbiome in recurrent Clostridium difficileassociated diarrhea, The Journal of Infectious Diseases 197 (2008) 435-438. DOI: $10.1086 / 525047$

Received: 18.12 .2018 Received in revised form: 06.03.2019 Accepted: 07.03.2019 